University of Nebraska - Lincoln

DigitalCommons@University of Nebraska - Lincoln

U.S. Environmental Protection Agency Papers

U.S. Environmental Protection Agency

2003

Evaluation of an alternative IMS dissociation procedure for use with Method 1622: detection of Cryptosporidium in water

Michael W. Ware

U.S. EPA, ware.michael@epa.gov

Larry Wymer

U.S. EPA

Alan Lindquist

U.S. EPA

Frank W. Schaefer III

U.S. EPA

Follow this and additional works at: https://digitalcommons.unl.edu/usepapapers

Ware, Michael W.; Wymer, Larry; Lindquist, Alan; and Schaefer, Frank W. III, "Evaluation of an alternative IMS dissociation procedure for use with Method 1622: detection of Cryptosporidium in water" (2003). U.S. Environmental Protection Agency Papers. 185.

https://digitalcommons.unl.edu/usepapapers/185

This Article is brought to you for free and open access by the U.S. Environmental Protection Agency at DigitalCommons@University of Nebraska - Lincoln. It has been accepted for inclusion in U.S. Environmental Protection Agency Papers by an authorized administrator of DigitalCommons@University of Nebraska - Lincoln. 


\section{Journal ${ }^{\text {of Microbiological }}$ Methods}

www.elsevier.com/locate/jmicmeth

\title{
Evaluation of an alternative IMS dissociation procedure for use with Method 1622: detection of Cryptosporidium in water
}

\author{
Michael W. Ware*, Larry Wymer, H.D. Alan Lindquist, Frank W. Schaefer III \\ National Exposure Research Laboratory, U.S.EPA, 26 W. Martin Luther King Dr., Cincinnati, OH 45268-1320, USA
}

Received 25 February 2003; received in revised form 23 June 2003; accepted 24 June 2003

\begin{abstract}
U.S.EPA Methods 1622 and 1623 are used to detect and quantify Cryptosporidium oocysts in water. The protocol consists of filtration, immunomagnetic separation (IMS), staining with a fluorescent antibody, and microscopic analysis. Microscopic analysis includes detection by fluorescent antibody and confirmation by the demonstration of 1-4 sporozoites or nuclei after staining with 4',6-diamidino-2-phenyl indole dihydrochloride (DAPI). The purpose of this study was to evaluate a new IMS dissociation, a 10 -min incubation at $80{ }^{\circ} \mathrm{C}$. Heat dissociation improved the average oocyst recovery from $41 \%$ to $71 \%$ in seeded reagent water, and from $10 \%$ to $51 \%$ in seeded river samples. The average DAPI confirmation rate improved from $49 \%$ to $93 \%$ in reagent water, and from $48 \%$ to $73 \%$ in river samples. This modification improved both oocyst recovery and confirmation. Published by Elsevier B.V.
\end{abstract}

Keywords: Cryptosporidium; Cytometry; DAPI; IMS; Method 1623; Oocyst

\section{Introduction}

Numerous waterborne outbreaks of cryptosporidiosis illustrate that Cryptosporidium oocysts in water pose a potential public health risk (Mac Kenzie et al., 1994; Atherton et al., 1995). The current benchmark in the United States for the detection of Cryptosporidium oocysts in water is U.S.EPA Methods 1622 or 1623 , both consisting of filtration, elution, purification by immunomagnetic separation (IMS), microscopic analysis after staining with a fluorescein isothiocyanate (FITC) conjugated monoclonal antibody (FAb) and counterstaining with 4',6-diamidino-2-phenyl-indole (DAPI) (U.S.EPA, 1999a,b; 2001a,b). Some

\footnotetext{
* Corresponding author. Tel.: +1-513-569-7731; fax: +1-513569-7117.

E-mail address: ware.michael@epa.gov (M.W. Ware).
}

algae either exhibit autofluorescence, or cross react with commercially available antibodies yielding false positives (Rodgers et al., 1995). Thus, oocyst-like objects identified by FAb staining must be confirmed by another method.

Oocysts detected by FAb in Method 1622 or 1623 must be confirmed by microscopic detection of 1-4 sporozoites or nuclei characteristic of Cryptosporidium. Sporozoites, and sometimes nuclei, may be detected by differential interference contrast microscopy (DIC), which is technically difficult and results in few confirmations, even in highly purified, fresh oocyst preparations (Lindquist et al., 2001). Nuclei of Cryptosporidium oocysts are more easily detected after staining with DAPI using epifluorescent microscopy. When DAPI staining is performed as described in Method 1623, the rate of oocyst confirmation is greater than by DIC sporozoite identifica- 
tion, but is still low. The DAPI staining procedure used was described by Grimason et al. (1994) except the oocyst in this study were not exposed to diethylether, due to safety concerns. The differences in oocyst staining between the two studies may be explained by this.

In a study comparing various detection methods (Lindquist et al., 2001), a solid phase cytometer protocol (Reynolds et al., 1999) had the highest DAPI confirmation rate of oocysts identified by $\mathrm{FAb}$ when compared to the other detection methods evaluated. This DAPI staining protocol incorporated heating oocysts for $10 \mathrm{~min}$ at $80{ }^{\circ} \mathrm{C}$ in the presence of $48 \%$ ethanol $(\mathrm{v} / \mathrm{v})$ prior to staining. In the present study, we also demonstrated that heating oocysts to $80{ }^{\circ} \mathrm{C}$ for $10 \mathrm{~min}$ before staining increases the confirmation rate by DAPI. We believe that the heat increases permeability of the oocyst cell wall. However, this adaptation has not been applied to Method 1622 or 1623 . We also found that heat dissociated the paramagnetic IMS bead-oocyst complex, and could replace the acid dissociation step described in Method 1622 or 1623 . A series of reagent and river water IMS samples were seeded with flow cytometry counted Cryptosporidium parvum oocysts. Oocyst recovery and confirmation rates were compared after acid dissociation followed by heating, or by heat dissociation alone.

\section{Materials and methods}

\subsection{Oocysts}

C. parvum (Harley Moon strain) oocysts were obtained from C. Sterling, University of Arizona, and were propagated in C57BL mice. They were purified by sieving, step sucrose gradients, and cesium chloride purification (Cicmanec and Reasoner, 1997). Oocysts were used within 3 months of purification. Oocysts were stored at $4{ }^{\circ} \mathrm{C}$, suspended in reagent grade water containing $100 \mathrm{U}$ of penicillin and $100 \mu \mathrm{g}$ streptomycin $/ \mathrm{ml}$.

\subsection{Staining and microscopy}

All oocysts were stained with $1 \times$ Crypt-a-glo or Aqua-glo (Waterborne, New Orleans, LA) and coun- terstained with DAPI (Sigma, St. Louis, MO) at 0.4 $\mu \mathrm{g} / \mathrm{ml}$, as described in Method 1623. Stained oocysts were identified using the criteria in Method 1623 (U.S.EPA, 2001b). Microscopic examinations were performed with a Zeiss Axiphot2 epifluorescence microscope (Carl Zeiss, Oberkochen, Germany) equipped with bright field, phase contrast, DIC, and epifluorescence optics. The primary FITC conjugated-antibody stained oocysts were observed with the following epifluorescence filter set: exciter filter, 450-490 nm; dichroic beam splitting, 510 $\mathrm{nm}$; barrier filter, $515-520 \mathrm{~nm}$. The DAPI stained nuclei were observed with the following epifluorescence filter set: exciter filter, $340-380 \mathrm{~nm}$; dichroic beam splitting, $400 \mathrm{~nm}$; barrier filter, $420 \mathrm{~nm}$. Photomicrographs were taken using a SPOT color digital camera (Diagnostic Instruments, Sterling Heights, MI).

\subsection{Flow cytometry}

A FACS VantageSE (Beckton Dickinson, Palo Alto, CA) equipped with CloneCyt software was used for all flow cytometry analysis. Isoton II (Coulter, Hiahlea, FL) was the sheath fluid. A primary gate was drawn around the oocysts using forward (FSC) and side scatter (SSC) with both FSC and SSC measured linearly with a gain of 1, SSC detector set at $399 \mathrm{~V}$, and FSC threshold was set at $32 \mathrm{~V}$. Oocysts that entered the FSC/SSC gate were analyzed on a plot of FL1 (FITC; illumination $488 \mathrm{~nm}$, bandpass filter 530/30 nm) and FL5 (DAPI; illumination 351-364 $\mathrm{nm}$ (UV), band pass filter 424/44 nm). Both fluorescent channels were measured in $\log$ mode with the FL1 detector set at $363 \mathrm{~V}$ and the FL5 detector set at $599 \mathrm{~V}$. The data from at least 1000 oocysts was used for all analysis.

Oocysts were prepared for experimental manipulations by sorting highly purified, unstained oocysts using the primary gate into $1.5 \mathrm{ml}$ tubes containing 1 $\mathrm{ml}$ reagent water.

\subsection{DAPI analysis}

C. parvum oocysts suspended in reagent water were used to compare two DAPI staining protocols. The first protocol was a control of oocysts suspended in $100 \mu \mathrm{l}$. The second was also suspended in $100 \mu \mathrm{l}$, 
but was then heated for $10 \mathrm{~min}$ in an $80{ }^{\circ} \mathrm{C}$ hot block, after Reynolds et al. (1999). Both samples were then stained by FAb and DAPI and examined by both flow cytometry and microscopy.

\section{5. $p H$ effect}

Replicates of a $3-\mu 1$ aliquot containing over 5000 oocysts were suspended in $50 \mu 1$ of $0.1,0.05,0.01$, 0.005 , and $0.001 \mathrm{~N} \mathrm{HCl}$ or $\mathrm{NaOH}$. All of these samples were heated for $10 \mathrm{~min}$ at $80{ }^{\circ} \mathrm{C}$, stained in suspension, and analyzed by flow cytometry. Heated and unheated oocysts suspended in reagent water served as controls.

\subsection{IMS experiments}

IMS experiments were performed in both reagent and river waters to compare two dissociation methods. Several grab samples $(>3001)$ were taken from the Ohio River on different days, filtered with Pall-Gelman Envirochek ${ }^{\circledR}$ filters, and eluted as described in Method 1623. These samples were pelleted, pooled into one large packed pellet, and suspended in $270 \mathrm{ml}$ reagent water (U.S.EPA, 1999a). The reagent water samples were not filtered and eluted.

For all IMS reactions, Dynabeads anti-Cryptosporidium kits (Dynal, Oslo, Norway) were used. Each $10 \mathrm{ml}$ IMS was composed of $1 \mathrm{ml}$ of an oocyst suspension, containing $150,100,50,20$, or 0 flow cytometry enumerated $C$. parvum oocysts in reagent water, $1 \mathrm{ml}$ of reagent water used to rinse the tube, and either $8 \mathrm{ml}$ of reagent water, or $4 \mathrm{ml}$ of the river water suspension and $4 \mathrm{ml}$ of reagent water. The 4 $\mathrm{ml}$ of river water suspension represented approximately $0.4 \mathrm{ml}$ of packed pellet. The IMS reactions were paired to compare the two dissociation procedures. The IMS reagents were added and processed as described in Method 1623 through the aspiration of the supernatant after the second IMS separation, step13.3.2.16 (U.S.EPA, 1999b).

One set was processed with acid dissociation, as described in Method 1623. Briefly, $50 \mu \mathrm{l}$ of $0.1 \mathrm{~N} \mathrm{HCl}$ was added to the oocyst-bead complex. This mixture was incubated for $10 \mathrm{~min}$ at room temperature, and the oocysts were separated from the paramagnetic beads by exposure to a magnetic strip. The oocysts in solution were transferred to another $1.5 \mathrm{ml}$ tube containing $5 \mu 11 \mathrm{~N} \mathrm{NaOH}$ and $50 \mu 1$ reagent water (U.S.EPA, 1999b). This tube was then heated for 10 min at $80{ }^{\circ} \mathrm{C}$ in a hot block.

One hundred microliters of reagent water was added to each tube in the other set. These oocysts were heated for $10 \mathrm{~min}$ at $80{ }^{\circ} \mathrm{C}$ in a hot block, separated from the paramagnetic beads by exposure to a magnetic strip, and transferred to another $1.5 \mathrm{ml}$ tube.

Both sets of tubes were then stained in solution, filtered through a $0.8-\mu \mathrm{m}$ porosity, $13 \mathrm{~mm}$ diameter polycarbonate filter, and enumerated by microscopy. For each water type, dissociation method, and oocyst seed level, at least five replicates were analyzed, with the exception of unseeded reagent water where only three replicates were performed for each dissociation method.

\subsection{Statistical analysis}

The percent of oocysts recovered and percent of those which were DAPI positive after acid dissociation were compared to the corresponding percent recovery and percent DAPI positive using heat dissociation via a multivariate analysis of variance (ANOVA). Because the relative recovery may vary depending on the number of oocysts in the sample, which ranged from 20 to 150 oocysts per sample, oocyst number was considered in the ANOVA as an additional factor. This enabled an evaluation of whether the relative difference between the two dissociation techniques varied by oocyst level (the method by oocyst level "interaction"). Separate analyses were performed for reagent water and environmental samples. The arcsine-square root of observed recovery proportion for each sample was used in the ANOVA, since these data represent proportions (Anderson and McLean, 1974).

Ideally, recoveries from different samples would be a simple Bernoulli, or binomial, process, in which the probability of oocyst recovery would be constant between samples. We evaluated the reasonableness of this binomial model by means of chi-square goodness-of-fit tests on each combination of dissociation method and type of sample (reagent water or river water). Lack of fit was an indication that $p$ varied from trial to trial, representing an additional source of variation in results beyond that of simple binomial 
outcomes. An example of a source of variation could be an undesigned $\mathrm{pH}$ change in one experimental group.

\section{Results}

\subsection{DAPI analysis}

Fig. 1 shows the FAb and DAPI staining flow cytometry profiles of two staining procedures. The unheated control had a very different DAPI profile compared to the heated oocysts. There was a close agreement between flow cytometry and microscopy for both protocols. The heated oocysts had a DAPI confirmation rate of at least $99 \%$ by either flow cytometry or microscopy. The control oocysts had a DAPI confirmation rate of $4 \%$ by microscopy and $2.3 \%$ by flow cytometry. The photomicrographs in Fig. 2 show FAb and DAPI stained C. parvum oocysts prepared by the two protocols. This figure shows same field images by FAb, DAPI, and DIC. In this figure all of the heated oocysts were DAPI positive while only a few of the control oocysts were DAPI positive. The FAb and DIC appearances are unchanged by heating.

\section{2. $p H$ effect}

The effect of $\mathrm{pH}$ was evaluated by suspending oocysts in various concentrations of $\mathrm{HCl}$ and $\mathrm{NaOH}$, followed by staining and examination by flow cytometry. Fig. 3 shows that FAb and DAPI stained oocysts are affected by small concentration changes in acid and base. The FAb signal intensity began to decrease when oocysts were suspended and stained in $0.005 \mathrm{~N}$ acid or base and was undetectable at higher concentrations. Although less sensitive to $\mathrm{pH}$ than FAb staining, DAPI signal intensity was adversely affected by increased concentrations of acid or base as well.

\subsection{IMS results}

Seeded reagent water samples were analyzed in parallel with either acid or heat dissociation. The results are shown in Table 1. The heat dissociated samples had significantly higher mean oocyst re-

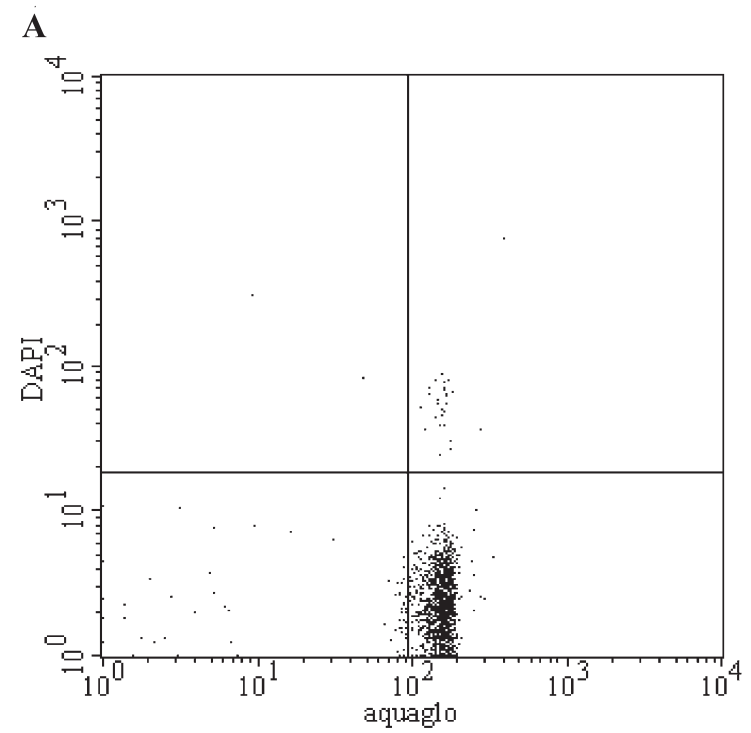

B

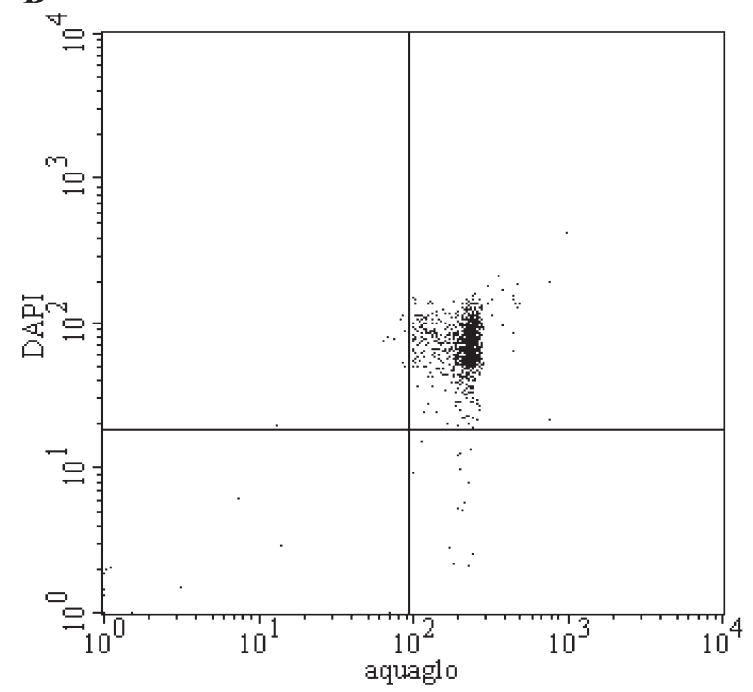

Fig. 1. Flow cytometric comparison of two C. parvum DAPI staining procedures. (A) C. parvum oocysts in reagent water and stained in suspension with Aqua-glo and DAPI. (B) C. parvum oocysts in reagent water, heated at $80{ }^{\circ} \mathrm{C}$ for $10 \mathrm{~min}$, and stained in suspension with Aqua-glo and DAPI.

covery rates when compared to the acid dissociated samples, $71 \%$ and $41 \%$, respectively $(p<0.0001)$. The heat dissociated samples also had significantly higher mean DAPI confirmation rates compared to acid dissociation, $93 \%$ and $49 \%$, respectively $(p \leq 0.0062)$. 

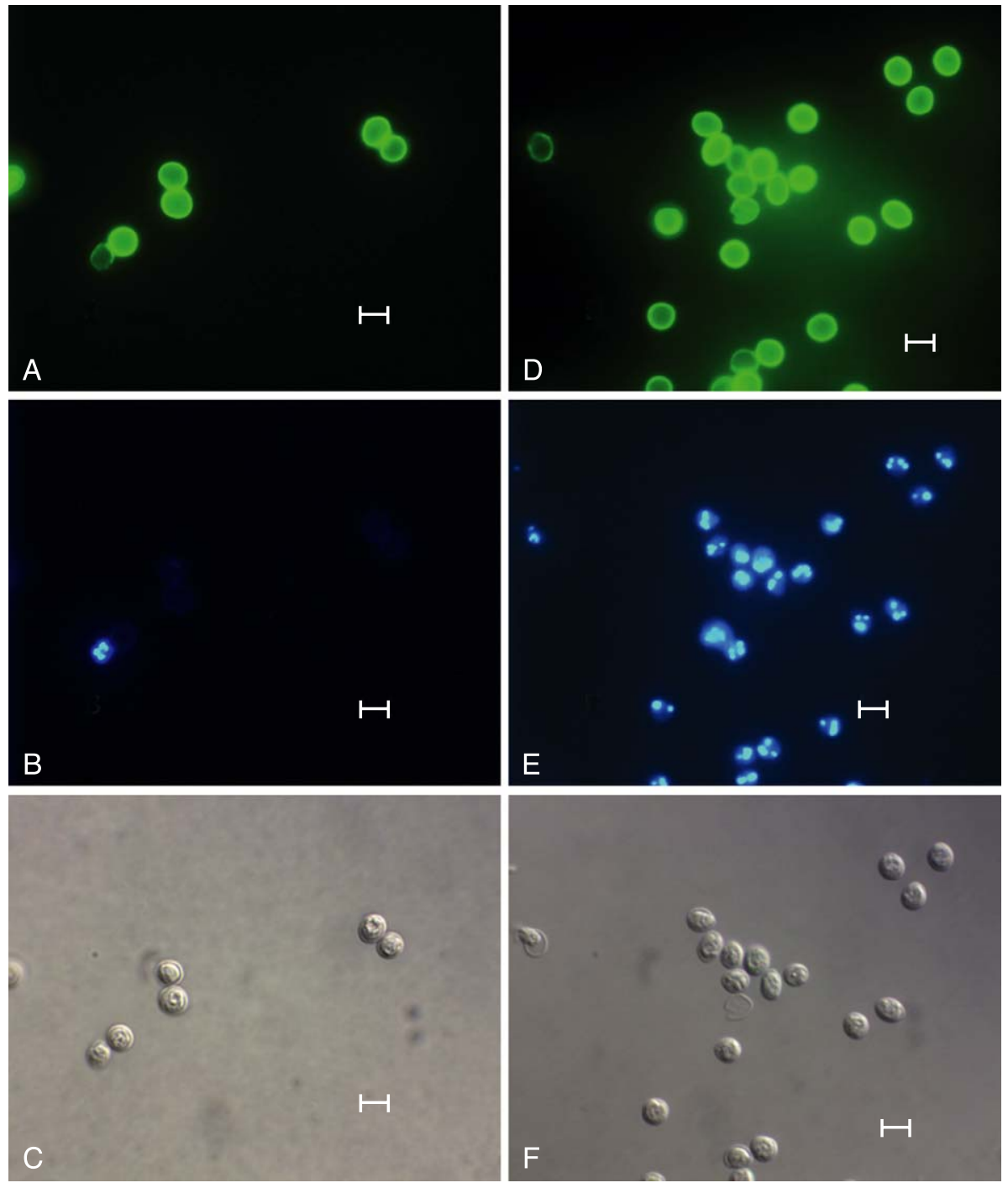

Fig. 2. Micrographs of control and heat treated DAPI stained C. parvum oocysts. A, B, and C: Same field micrographs of C. parvum oocysts stained by FAb and DAPI. (A) FAb stained. (B) DAPI stained. (C) DIC. D, E, and F: Same field micrographs of $C$. parvum oocysts stained by $\mathrm{FAb}$ and DAPI after $80{ }^{\circ} \mathrm{C}$ heat for $10 \mathrm{~min}$. (D) FAb stained. (E) DAPI stained. (F) DIC. Scale bar $=5 \mu \mathrm{m}$.

River water samples seeded with oocysts were analyzed in parallel with either acid or heat dissociation, and the results are shown in Table 2. The river water samples had lower mean recovery rates than in the reagent water samples. However, the mean oocyst recovery in the heat dissociated samples were significantly greater than the acid dissociated samples, $51 \%$ and $10 \%(p<0.0001)$. DAPI confirmation rates also significantly increased with heat dissociation when compared to acid dissociation, $73 \%$ and $48 \%$, respectively $(p \leq 0.0002)$. There were a few oocysts detected in some of the unseeded river water samples dissociated by heat; however, their concentration averaged 0.8 oocysts/ 101 and they were not used in data analysis. No oocysts were detected in the remaining unseeded 
A

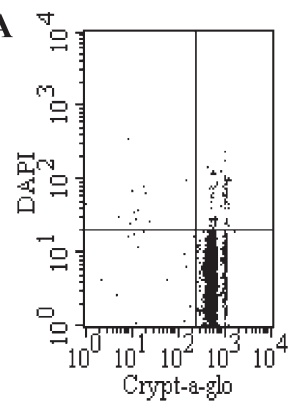

1
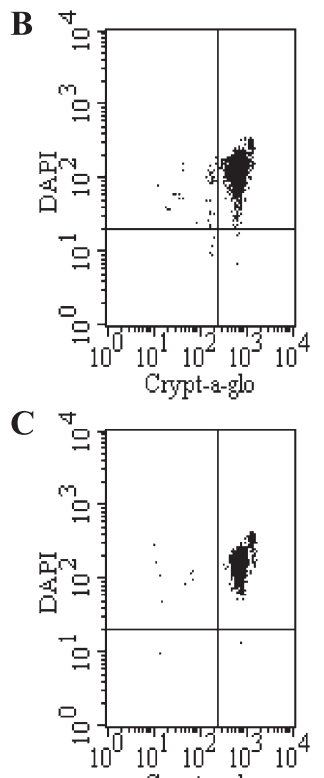

Crypt-a-glo
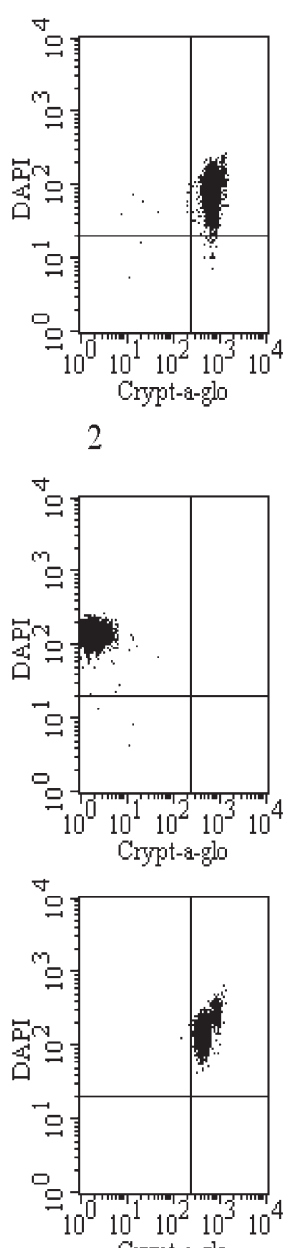

Crypt-a-glo
3
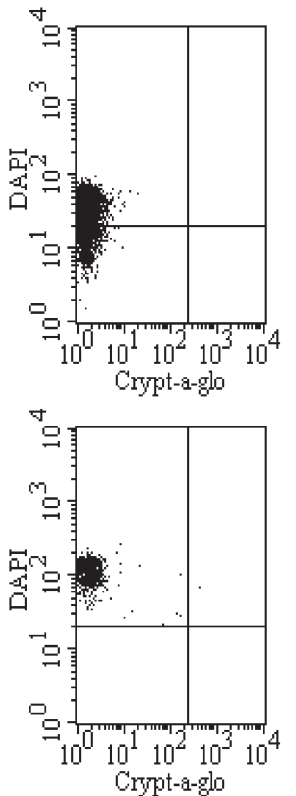

4
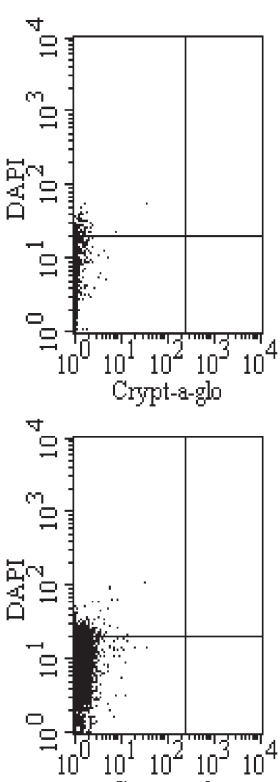

Crypt-a-glo

Fig. 3. Effect of $\mathrm{pH}$ on staining of $C$. parvum oocysts. All oocysts were stained in suspension by Crypt-a-glo and DAPI. (A) Oocysts suspended in reagent water. (1) Not heated. (2) Heated $80{ }^{\circ} \mathrm{C}$ for $10 \mathrm{~min}$. (B) Oocysts in concentrations of $\mathrm{HCl}$, and heated $80{ }^{\circ} \mathrm{C}$ for 10 min.: (1) $0.001 \mathrm{~N}$, (2) $0.005 \mathrm{~N}$, (3) $0.01 \mathrm{~N}$, (4) $0.05 \mathrm{~N}$, (5) $0.1 \mathrm{~N}$. (C) Oocysts in concentrations of $\mathrm{NaOH}$, and heated $80{ }^{\circ} \mathrm{C}$ for 10 min.: (1) $0.001 \mathrm{~N}$, (2) $0.005 \mathrm{~N}$, (3) $0.01 \mathrm{~N}$, (4) $0.05 \mathrm{~N}$, (5) $0.1 \mathrm{~N}$. 
Table 1

IMS recovery and DAPI confirmation rates of seeded $C$. parvum oocysts suspended in reagent water using acid and heat dissociations

\begin{tabular}{|c|c|c|c|c|c|}
\hline \multirow{2}{*}{$\begin{array}{l}\text { Number of } \\
\text { oocysts seeded }\end{array}$} & \multirow{2}{*}{$\begin{array}{l}\text { Number of } \\
\text { replicates }\end{array}$} & \multicolumn{2}{|c|}{ IMS with acid dissociation followed by heat } & \multicolumn{2}{|c|}{ IMS with heat dissociation } \\
\hline & & $\begin{array}{l}\text { \% Recovered } \\
\text { (Mean } \pm 2 \text { S.D.) }\end{array}$ & $\begin{array}{l}\% \text { DAPI positive } \\
\text { (Mean } \pm 2 \text { S.D.) }\end{array}$ & $\begin{array}{l}\text { \% Recovered } \\
\text { (Mean } \pm 2 \text { S.D.) }\end{array}$ & $\begin{array}{l}\% \text { DAPI positive } \\
\text { (Mean } \pm 2 \text { S.D.) }\end{array}$ \\
\hline 150 & 5 & $25 \pm 62.6$ & $51 \pm 96.4$ & $71 \pm 12.0$ & $92 \pm 13.4$ \\
\hline 100 & 7 & $30 \pm 58.2$ & $46 \pm 92.4$ & $74 \pm 15.6$ & $93 \pm 13.6$ \\
\hline 50 & 5 & $52 \pm 22.2$ & $51 \pm 69.4$ & $69 \pm 13.4$ & $90 \pm 6.0$ \\
\hline 20 & 11 & $51 \pm 60.0$ & $48 \pm 66.8$ & $70 \pm 27.2$ & $94 \pm 7.8$ \\
\hline Average & 28 & $41 \pm 57.4$ & $49 \pm 72.6$ & $71 \pm 19.8$ & $93 \pm 10.2$ \\
\hline 0 & 3 & $0 \pm 0$ & $0 \pm 0$ & $0 \pm 0$ & $0 \pm 0$ \\
\hline
\end{tabular}

All comparisons between the two methods are significant at $p<0.01$.

samples. Preliminary results using heat dissociation with Method 1623 indicate that the recovery and confirmation of Giardia lamblia cysts is also improved when compared to acid dissociation (data not shown).

Relative differences in recovery between the two dissociation methods did not differ significantly by the number of oocysts that were present, in either reagent or river water samples. The variation in recovery of heat dissociated oocysts was consistent with a binomial process meaning there was a constant probability of recovery from sample to sample, true in both reagent water $(p=0.93)$ and river water $(p=0.34)$ samples. On the other hand, there was significant lack of fit to a binomial process for acid dissociation ( $p<0.0001$ for both matrices), indicating that the probability of recovering an oocyst varied from one sample to the next. This suggests that some external source of variation affected recovery with acid dissociation.

\section{Discussion}

The existence of water matrix particulates such as algae require that Cryptosporidium oocyst-like objects detected by FAb be confirmed by some other method to prevent false positives. The classic confirmation method is by the demonstration of 1-4 sporozoites in an oocyst using DIC microscopy. Very few oocysts are confirmed by DIC even from fresh, highly purified preparations. DAPI staining of the nuclei as described in Method 1623 improves the rate of confirmation from that achieved by using DIC, however, many oocysts still cannot be confirmed (Lindquist et al., 2001).

Heating oocysts before DAPI staining increased the rate of DAPI confirmation in both pure preparations and river water samples. These DAPI stained oocysts were detectable by flow cytometry, and the results of flow cytometric analysis were in agreement with those obtained by microscopy. This study

Table 2

IMS recovery and DAPI confirmation rates of seeded $C$. parvum oocysts suspended in river water using acid and heat dissociations

\begin{tabular}{|c|c|c|c|c|c|}
\hline \multirow{2}{*}{$\begin{array}{l}\text { Number of } \\
\text { oocysts seeded }\end{array}$} & \multirow{2}{*}{$\begin{array}{l}\text { Number of } \\
\text { replicates }\end{array}$} & \multicolumn{2}{|c|}{ IMS with acid dissociation followed by heat } & \multicolumn{2}{|c|}{ IMS with heat dissociation } \\
\hline & & $\begin{array}{l}\text { \% Recovered } \\
\text { (Mean } \pm 2 \text { S.D.) }\end{array}$ & $\begin{array}{l}\% \text { DAPI positive } \\
\text { (Mean } \pm 2 \text { S.D.) }\end{array}$ & $\begin{array}{l}\text { \% Recovered } \\
(\text { Mean } \pm 2 \text { S.D.) }\end{array}$ & $\begin{array}{l}\% \text { DAPI positive } \\
\text { (Mean } \pm 2 \text { S.D.) }\end{array}$ \\
\hline 150 & 5 & $7 \pm 24.8$ & $57 \pm 56.4$ & $47 \pm 17.2$ & $82 \pm 6.2$ \\
\hline 100 & 5 & $12 \pm 28.0$ & $58 \pm 35.4$ & $60 \pm 16.4$ & $73 \pm 6.0$ \\
\hline 50 & 5 & $9 \pm 26.8$ & $40 \pm 36.0$ & $54 \pm 12.0$ & $76 \pm 13.6$ \\
\hline 20 & 5 & $14 \pm 27.8$ & $29 \pm 68.4$ & $43 \pm 23.0$ & $62 \pm 38.0$ \\
\hline Average & 20 & $10 \pm 25.4$ & $48 \pm 49.4$ & $51 \pm 20.8$ & $73 \pm 23.8$ \\
\hline 0 & 5 & $0 \pm 0$ & $0 \pm 0$ & $0.8 \pm 2.2$ & $25.0 \pm 70.8$ \\
\hline
\end{tabular}

All comparisons between the two methods are significant at $p<0.01$. 
demonstrates the utility of flow cytometry for the automated enumeration and confirmation of Cryptosporidium oocysts. Furthermore, because most oocysts now can be confirmed by DAPI stained nuclei, and at best only a few sporozoites are detected by DIC microscopy, DIC confirmation could be eliminated from Method 1623.

Acid dissociation in Method 1622 is achieved by pipetting of $50 \mu \mathrm{l}$ of $0.1 \mathrm{~N} \mathrm{HCl}$ and later $5 \mu \mathrm{l} \mathrm{N}$ $\mathrm{NaOH}$ resulting in a theoretical neutral pH (U.S.EPA, 1999b). Depending upon the matrix buffering capacity and pipetting accuracy, it is likely that the final $\mathrm{pH}$ fluctuates between samples. The FAb signal can virtually disappear when suspended in $0.005 \mathrm{~N}$ acid or base. Small changes in $\mathrm{pH}$ away from neutral may have been responsible for the low recovery and variable results reported in previous studies evaluating Methods 1622 and 1623 (Lindquist et al., 2001; Simmons et al., 2001a,b; DiGiorgio et al., 2002). A study evaluating the effect of $\mathrm{pH}$ during IMS reactions demonstrated that the reactions adjusted to $\mathrm{pH}$ 7.5 had higher mean recoveries than unadjusted IMS reactions (Kuhn et al., 2002). There are several possible explanations for the $\mathrm{pH}$ effect, including a decrease in antibody avidity or fluorochrome expression (Shapiro, 1995). Heat dissociation eliminated the potential $\mathrm{pH}$ variation associated with acid dissociation.

For both river and reagent water samples, the oocyst recovery was significantly greater in the samples processed with heat dissociation than with acid. However, the recoveries achieved in reagent water were greater than those observed in river water. This may be explained by a combination of $\mathrm{pH}$ buffer effects, debris, and higher background fluorescence levels in river water samples. The weaker FAb oocyst signal at a non-neutral $\mathrm{pH}$ may have been overwhelmed by natural fluorescence or debris found in river water. Reagent water had virtually no background fluorescence and therefore weaker fluorescent signals were more readily detected.

In summary, modification of Method 1622 by changing the IMS dissociation procedure to heat would improve oocyst recovery, improve DAPI staining, and reduce the variation seen in results. Flow cytometry also could be a useful tool for the automated analysis of samples. These results were verified by the analysis of real water samples.

\section{References}

Anderson, V.L., McLean, R.A., 1974. Design of Experiments. Marcel Drekker, New York, NY.

Atherton, F., Newman, C.P.S., Casemore, D.P., 1995. An outbreak of waterborne cryptosporidiosis associated with a public water supply in the UK. Epidemiol. Infect. 115, 123-131.

Cicmanec, J.L., Reasoner, D.J., 1997. Enhanced production of Cryptosporidium parvum oocysts in immunosuppressed mice. Proc. International Symposium on Waterborne Cryptosporidium, March, 1997. Denver, CO.

DiGiorgio, C.L., Gonzalez, D.A., Huitt, C.C., 2002. Cryptosporidium and Giardia recoveries in natural waters by using Environmental Protection Agency Method 1623. Appl. Environ. Microbiol. 68, 5952-5955.

Grimason, A.M., Smith, H.V., Parker, J.F.W., Bukhari, Z., Campbell, A.T., Robertson, L.J., 1994. Application of DAPI and immunofluorescence for enhanced identification of Cryptosporidium spp. oocysts in water samples. Water Res. 28, $733-736$.

Kuhn, R.C., Rock, C.M., Oshima, K.H., 2002. Effects of pH and magnetic material on immunomagnetic separation of Cryptosporidium oocysts from concentrated water samples. Appl. Environ. Microbiol. 68, 2066-2070.

Lindquist, H.D.A., Ware, M.W., Stetler, R.E., Wymer, L., Schaefer III, F.W., 2001. A comparison of four fluorescent antibody based methods for purifying, detecting, and confirming Cryptosporidium parvum in surface waters. J. Parasitol. 87, 1124-1131.

Mac Kenzie, W.R., Hoxie, N.J., Proctor, M.E., Gradus, M.S., Blair, K.A., Peterson, D.E., Kazmierczak, J.J., Addiss, D.G., Fox, K.R., Rose, J.B., 1994. A massive outbreak in Milwaukee of Cryptosporidium infection transmitted through the public water supply. N. Engl. J. Med. 331, 161-167.

Reynolds, D.T., Slade, R.B., Sykes, N.J., Jonas, A., Fricker, C.R., 1999. Detection of Cryptosporidium oocysts in water: techniques for generating precise recovery data. J. Appl. Microbiol. 87, 804-813.

Rodgers, M.R., Flanigan, D.J., Jakubowski, W., 1995. Identification of algae which interfere with the detection of Giardia cysts and Cryptosporidium oocysts and a method for alleviating this interference. Appl. Environ. Microbiol. 61, 3759-3763.

Shapiro, H.M., 1995. Practical Flow Cytometry. Wiley-Liss, New York, NY.

Simmons III, O.D., Sobsey, M.D., Schaefer III, F.W., Francy, D.S., Nally, R.A., Heaney, C.D., 2001a. Concentration and detection of Cryptosporidium oocysts in surface water samples by Method 1622 using ultrafiltration and capsule filtration. Appl. Environ. Microbiol. 67, 1123-1127.

Simmons III, O.D., Sobsey, M.D., Schaefer III, F.W., Francy, D.S., Nally, R.A., Heaney, C.D., 2001b. Evaluation of U.S.EPA Method 1622 for detection of Cryptosporidium oocysts in stream waters. J. Amer. Water Works Assoc. 93, 78-87.

U.S.EPA, 1999a. Method 1622: Cryptosporidium in water by filtration/IMS/FA. United States Environmental Protection Agency. Office of Water, Washington, DC. EPA 821-R99-001.

U.S.EPA, 1999b. Method 1623: Giardia and Cryptosporidium in water by filtration/IMS/FA. United States Environmental 
Protection Agency. Office of Water, Washington, DC. EPA 821-R99-006.

U.S.EPA, 2001a. Method 1622: Cryptosporidium in water by filtration/IMS/FA. United States Environmental Protection Agency. Office of Water, Washington, DC. EPA 821-R01-026.
U.S.EPA, 2001b. Method 1623: Giardia and Cryptosporidium in water by filtration/IMS/FA. United States Environmental Protection Agency. Office of Water, Washington, DC. EPA 821R01-025. 